\title{
A Table of Integrals of the Exponential Integral ${ }^{*}$
}

\author{
Murray Geller** and Edward W. Ng** \\ (March 11, 1969)
}

\begin{abstract}
This is a compendium of indefinite and definite integrals of products of the Exponential Integral with elementary or transcendental functions. A substantial portion of the results are new.

Key words: Diffusion theory; exponential integral; indefinite integrals; quantum mechanics; radiative equilibrium; special functions; transport problems.
\end{abstract}

\section{Introduction}

Integrals of the exponential integral occur in a wide variety of applications. Examples of applications can be cited from diffusion theory [12], ${ }^{1}$ transport problems [12], the study of the radiative equilibrium of stellar atmospheres [9], and in the evaluation of exchange integrals occurring in quantum mechanics [11]. This paper is an attempt to give an up-to-date exhaustive tabulation of such integrals.

All formulas for indefinite integrals in section 4 were derived from integration by parts and checked by differentiation of the resulting expressions. The formulas given in $[1,4,5,6,7,10$, 14, and 15] have all been checked and included, with the omission of trivial duplications. Additional formulas were obtained either from the various integral representations, from the hypergeometric series for the exponential integrals, from multiple integrals involving elementary functions, from the existing literature $[2,3,12$, and 13$]$, or by specialization of parameters of integrals over confluent hypergeometric functions [4] and [6].

Throughout this paper, we have adhered to the notations used in the NBS Handbook [8] and we have also assumed the reader's familiarity with the properties of the exponential integral. In addition, the reader should also attend to the following conventions:

(i) The integration constants have been omitted for the indefinite integrals;

(ii) the parameters $a, b$, and $c$ are real and positive except where otherwise stated;

(iii) unless otherwise specified, the parameters $n$ and $k$ represent the integers $0,1,2 \ldots$, whereas the parameters $p, q$, and $\mu$ and $\nu$ may be nonintegral;

(iv) the integration symbol $f$ denotes a Cauchy principal value;

(v) $x, y$, and $t$ represent real variables.

\section{Glossary of Functions and Notations}

ber $(x)$, bei $(x)$

$B_{x}(p, q)$

$\operatorname{Ci}(x)$

$e_{n}(x)$
Thomson functions

Incomplete beta function

Cosine integral

Truncated exponential $\int_{0}^{x} t^{p-1}(1-t)^{q-1} d t$

$-\int_{x}^{\infty} \frac{\cos t}{t} d t$

$\sum_{m=0}^{n} \frac{x^{m}}{m !}$

*An invited paper. This paper presents the results of one phase of research carried out at the Jet Propulsion Laboratory, California Institute of Technology, under Contract No. NAS7-100 sponsored by the National Aeronautics and Space Administration.

**Present address: Jet Propulsion Laboratory, California Institute of Technology, Pasadena, Calif. 91103.

${ }^{1}$ Figures in brackets indicate the literature references at the end of this paper. 


\begin{tabular}{|c|c|c|}
\hline$E_{1}(x)$ & Exponential integral & $\int_{x}^{\infty} \frac{e^{-t}}{t} d t$ \\
\hline$E i(x)$ & Exponential integral & $-\int_{-x}^{\infty} \frac{e^{-t}}{t} d t$ \\
\hline $\operatorname{erf}(x)$ & Error function & $\frac{2}{\sqrt{\pi}} \int_{0}^{x} e^{-t^{2}} d t$ \\
\hline $\operatorname{erfc}(x)$ & Error function & $\frac{2}{\sqrt{\pi}} \int_{x}^{\infty} e^{-t^{2}} d t$ \\
\hline${ }_{1} F_{1}(a ; b ; x)$ & Confluent hypergeometric function & $\sum_{n=0}^{\infty} \frac{(a)_{n}}{(b)_{n}} \cdot \frac{x^{n}}{n !}$ \\
\hline${ }_{2} F_{1}(a, b ; c ; x)$ & Hypergeometric function & $\sum_{n=0}^{\infty} \frac{(a)_{n}(b)_{n}}{(c)_{n}} \cdot \frac{x^{n}}{n !}$ \\
\hline $\begin{array}{l}{ }_{p} \boldsymbol{F}_{q} \\
\mathbf{H}_{p}(x) \\
I_{p}(x) \\
J_{p}(x) \\
K_{p}(x)\end{array}$ & $\begin{array}{l}\text { Generalized hypergeometric function } \\
\text { Struve function } \\
\text { Bessel function of imaginary argument } \\
\text { Bessel function } \\
\text { Bessel function of imaginary argument }\end{array}$ & \\
\hline $\operatorname{li}(x)$ & Logarithmic integral & $f_{0}^{x} \frac{d t}{\ln t}, x>1$ \\
\hline$L_{2}(x)$ & Euler's dilogarithm & $-\int_{0}^{x} \frac{\ln (1-t)}{t} d t$ \\
\hline$L_{n}(x)$ & Laguerre polynomial & $\frac{e^{x}}{n !} \frac{d^{n}}{d x^{n}}\left(x^{n} e^{-x}\right)$ \\
\hline $\begin{array}{l}M_{p, q}(x) \\
\left(\begin{array}{c}m \\
n\end{array}\right)\end{array}$ & $\begin{array}{l}\text { Whittaker function } \\
\text { Binomial coefficient }\end{array}$ & $\frac{m !}{n !(m-n) !}$ \\
\hline$P_{\nu}^{\mu}(x)$ & Associated Legendre function of the first kind & \\
\hline$(p)_{n}$ & Pochhammer's symbol & $\frac{\Gamma(p+n)}{\Gamma(p)}$ \\
\hline $\operatorname{si}(x)$ & Sine integral & $-\int_{x}^{\infty} \frac{\sin t}{t} d t$ \\
\hline $\begin{array}{l}\operatorname{sgn} x \\
W_{p, q}(x) \\
Y_{p}(x)\end{array}$ & $\begin{array}{l}\text { Sign of the real number } \\
\text { Whittaker function } \\
\text { Neumann function }\end{array}$ & $x /|x|$ \\
\hline$\gamma$ & Euler's constant & $0.57721 \quad 56649$ \\
\hline$\gamma(a, x)$ & Incomplete gamma function & $\int_{0}^{x} e^{-t} t^{a-1} d t$ \\
\hline$\Gamma(a, x)$ & Incomplete gamma function & $\int_{x}^{\infty} e^{-t} t^{a-1} d t$ \\
\hline$\Gamma(a)$ & Gamma function & $\int_{0}^{\infty} e^{-t} t^{a-1} d t$ \\
\hline$\zeta(p)$ & Riemann zeta function & $\sum_{m=0}^{\infty} \frac{1}{(m+1)^{P}}$ \\
\hline
\end{tabular}


Confluent hypergeometric function.

\section{Definition, Special Values, and Integral Representations}

\subsection{Definition and Other Notations}

1. $E_{1}(x)=\int_{x}^{\infty} \frac{e^{-t}}{t} d t \quad x>0$

2. $\operatorname{Ei}(x)=-f_{-x}^{\infty} \frac{e^{-t}}{t} d t$

$$
=f_{-\infty}^{x} \frac{e^{t}}{t} d t \quad x>0 .
$$

3. Some authors use $[-E i(-x)]$ for $E_{1}(x)$.

4. Some authors use $E^{*}(x)$ or $\bar{E} i(x)$ for $E i(x)$.

5. Integrals involving $l i(x)$ can be transformed into integrals over $E i(x)$ since

$$
\operatorname{li}(x)=f_{0}^{x} \frac{d t}{\ln t}=E i(\ln x) \quad x>1 .
$$

\subsection{Special Values}

1. $E i(0.372507 \ldots)=0$.

2. $E i(x)=E_{1}(x)$ at $x=0.523823 \ldots$

3. $\operatorname{Lim}_{x \rightarrow 0}\left[x^{p} E_{1}(x)\right]=\operatorname{Lim}_{x \rightarrow 0}\left(x^{p} E i(x)\right)=0 \quad p>0$.

4. $\operatorname{Lim}_{x \rightarrow 0}\left[\ln x+E_{1}(x)\right]=-\gamma$.

5. $\operatorname{Lim}_{x \rightarrow \infty}\left[x^{p} E_{1}(x)\right]=\operatorname{Lim}_{x \rightarrow \infty}\left[\ln x E_{1}(x)\right]=0$.

6. $\operatorname{Lim}_{x \rightarrow \infty}\left[e^{-x} E i(x)\right]=0$.

7. Inflection point of $E i(x)$ at $x=1$.

\subsection{Integral Representations}

1. $E_{1}(x)=-\gamma-\ln x+\int_{0}^{x}\left(1-e^{-t}\right) \frac{d t}{t}$

2. $E_{1}(x)=-\gamma-e^{-x} \ln x-\int_{0}^{x} e^{-t} \ln t d t$

3. $E_{1}(x)=x \int_{1}^{\infty} e^{-x t} \ln t d t$

4. $E_{1}(x)=e^{-x} \int_{0}^{1} \frac{1}{(x-\ln t)} d t$

5. $E_{1}(x)=e^{-x} \int_{1}^{\infty} \frac{1}{(x+\ln t)} \frac{d t}{t^{2}}$ 
6. $E_{1}(x)=e^{-x} \int_{0}^{\infty} \frac{e^{-x t}}{(1+t)} d t$

7. $E_{1}(x)=\frac{e^{-x}}{x}\left[1-\int_{0}^{\infty} \frac{x}{(x+t)^{2}} e^{-t} d t\right]$

8. $E_{1}(x)=\frac{e^{-x}}{x}\left[1-\int_{0}^{1} \frac{t^{x-1}}{(1-\ln t)^{2}} d t\right]$

9. $E_{1}(x)=e^{-x} \int_{0}^{\infty} \ln \left(1+\frac{t}{x}\right) e^{-t} d t$

10. $E_{1}(x)=\frac{1}{\pi} \int_{0}^{\infty} \sin t \ln \left(1+\frac{t^{2}}{x^{2}}\right) \frac{d t}{t}$

11. $E_{1}(x)=2 e^{-x} \int_{0}^{\infty} K_{0}(2 \sqrt{x t}) e^{-t} d t$

12. $E_{1}(x)=e^{-x} \int_{0}^{\infty} \frac{1}{(t-i x)} e^{-i t} d t$

13. $E_{1}(x)=\int_{0}^{\infty} \exp \left(-x e^{t}\right) d t$

14. $E i(x)=e^{x} \int_{0}^{1} \frac{1}{(x+\ln t)} d t$

15. $E i(x)=e^{x} \int_{1}^{\infty} \frac{1}{(x-\ln t)} \frac{d t}{t^{2}}$

16. $E i(x)=e^{x} \int_{0}^{\infty} \frac{e^{-x t}}{(1-t)} d t$

17. $E i(x)=\frac{e^{x}}{x}\left[\int_{0}^{\infty} \frac{x e^{-t}}{(x-t)^{2}} d t+1\right]$

18. $E i(x)=\frac{e^{x}}{x}\left[\int_{0}^{1} \frac{t^{x-1}}{(1+\ln t)^{2}} d t+1\right]$

19. $E i(x)=-e^{x} \int_{0}^{\infty} \frac{e^{-i t}}{(t+i x)} d t$

20. $\left[E_{1}(x)\right]^{2}=2 e^{-x} \int_{1}^{\infty} \frac{e^{-x t}}{(1+t)} \ln t d t$.

21. $E_{1}(a x) E_{1}(b x)+E_{1}[(a+b) x] \ln (a b)=e^{-(a+b) x} \int_{0}^{\infty} \frac{e^{-x t}}{(a+b+t)} \ln [(a+t)(b+t)] d t$

22. $e^{-x} E i(x)+e^{x} E_{1}(x)=2 \int_{0}^{\infty} \frac{x}{\left(t^{2}+x^{2}\right)} \sin t d t$

23. $e^{-x} \operatorname{Ei}(x)+e^{x} E_{1}(x)=2 e^{-x} \ln x-\frac{4}{\pi} \int_{0}^{\infty} \frac{x}{\left(t^{2}+x^{2}\right)} \cos t \ln t d t$

24. $e^{-x} E i(x)-e^{x} E_{1}(x)=-2 \int_{0}^{\infty} \frac{t}{\left(t^{2}+x^{2}\right)} \cos t d t$

25. $e^{-x} \operatorname{Ei}(x)-e^{x} E_{1}(x)=2 e^{-x} \ln x-\frac{4}{\pi} \int_{0}^{\infty} \frac{t}{\left(t^{2}+x^{2}\right)} \sin t \ln t d t$ 


\section{Integrals of the Exponential Integral With Other Functions}

\subsection{Combination of Exponential Integral With Powers}

1. $\int E_{1}(a x) d x=x E_{1}(a x)-\frac{1}{a} e^{-a x}$

2. $\int E i(a x) d x=x E i(a x)-\frac{1}{a} e^{a x}$

3. $\int_{0}^{\infty} E_{1}(a x) d x=\frac{1}{a}$

4. $\int x E_{1}(a x) d x=\frac{1}{2} x^{2} E_{1}(a x)-\frac{1}{2 a^{2}}(1+a x) e^{-a x}$

5. $\int_{0}^{\infty} x E_{1}(a x) d x=\frac{1}{2 a^{2}}$

6. $\int x^{n} E_{1}(a x) d x=\frac{x^{n+1}}{(n+1)} E_{1}(a x)-\frac{n !}{(n+1)} \frac{1}{a^{n+1}} e_{n}(a x) e^{-a x}$

7. $\int x^{n} E_{1}(a x+b) d x=E_{1}(a x+b) \sum_{m=0}^{n}(-1)^{m} \frac{n !}{(n-m) !} \frac{x^{n-m}}{(m+1) !}\left(x+\frac{b}{a}\right)^{m+1}$

$$
-e^{-(a x+b)} \sum_{m=0}^{n} \frac{n !}{(n-m) !} \frac{1}{(m+1) !} \frac{x^{n-m}}{a^{m+1}} \sum_{k=0}^{m}(-1)^{k}(m-k) !(a x+b)^{k}
$$

8. $\int_{0}^{\infty} x^{n} E_{1}(a x) d x=\frac{n !}{(n+1)} \cdot \frac{1}{a^{n+1}}$

9. $\int_{0}^{\infty} x^{n} E_{1}(a x+b) d x=\frac{1}{(n+1)} \cdot \frac{1}{a^{n+1}}\left[(-1)^{n+1} b^{n+1} E_{1}(b)+\sum_{m=0}^{n}(-1)^{m} b^{m}(n-m) ! e^{-b}\right]$

10. $\int_{a}^{\infty} x^{n} E_{1}(x-y) d x=e^{-(a-y)} \sum_{m=0}^{n}\left(\begin{array}{l}n \\ m\end{array}\right) \frac{m !}{(m+1)} y^{n-m} e_{m}(a-y)$

$$
-E_{1}(a-y) \sum_{m=0}^{n}\left(\begin{array}{l}
n \\
m
\end{array}\right) \frac{y^{n-m}}{(m+1)}(a-y)^{m+1} \quad y<a
$$

11. $\int_{a}^{\infty} x^{n} E_{1}(x-a) d x=\sum_{m=0}^{n}\left(\begin{array}{l}n \\ m\end{array}\right) \frac{m !}{(m+1)} a^{n-m}$

12. $\int_{a}^{\infty} x^{n} E_{1}(|y-x|) d x=\sum_{m=0}^{n}\left(\begin{array}{l}n \\ m\end{array}\right) \frac{m !}{(m+1)}\left[1+(-1)^{m}\right] y^{n-m}$

$$
\begin{aligned}
& -e^{-(y-a)} \sum_{m=0}^{n}\left(\begin{array}{c}
n \\
m
\end{array}\right) \frac{m !}{(m+1)}(-1)^{m} y^{n-m} e_{m}(y-a) \\
& +E_{1}(y-a) \sum_{m=0}^{n}\left(\begin{array}{c}
n \\
m
\end{array}\right) \frac{(-1)^{m}}{(m+1)} y^{n-m}(y-a)^{m+1} \quad y>a
\end{aligned}
$$

13. $\int_{0}^{\infty} x^{n} E_{1}(|y-x|) d x=\sum_{m=0}^{n}\left(\begin{array}{l}n \\ m\end{array}\right) \frac{m !}{(m+1)}\left[1+(-1)^{m}\right] y^{n-m}$

$$
-e^{-y} \sum_{m=0}^{n}\left(\begin{array}{l}
n \\
m
\end{array}\right) \frac{m !}{(m+1)}(-1)^{m} y^{n-m} e_{m}(y)+\frac{y^{n+1}}{(n+1)} E_{1}(y)
$$


14. $\int x^{p} E_{1}(a x) d x=\frac{x^{p+1}}{(p+1)} E_{1}(a x)+\frac{1}{(p+1)} \frac{1}{a^{p+1}} \gamma(p+1, a x) \quad p>-1$

15. $\int_{0}^{\infty} x^{p} E_{1}(a x) d x=\frac{1}{(p+1)} \frac{1}{a^{p+1}} \Gamma(p+1) \quad p>-1$

16. $\int_{0}^{\infty} x^{p} E_{1}(x+b) d x=\Gamma(p+1) e^{-b / 2} b^{p / 2} W_{\dashv(p+2) / 2,-(p+1) / 2}(b) \quad p>-1$

17. $\int E_{1}(a x) \frac{d x}{x}=\int e^{-a x} \ln x \frac{d x}{x}+\ln x E_{1}(a x)$

18. $\int_{b}^{\infty} E_{1}(a x) \frac{d x}{x}=\frac{1}{2}\left[(\gamma+\ln a b)^{2}+\zeta(2)\right]+\sum_{n=1}^{\infty} \frac{(-a b)^{n}}{n ! n^{2}}$

19. $\int_{b}^{\infty} E_{1}(x+b) \frac{d x}{x}=\frac{1}{2}\left[E_{1}(b)\right]^{2}$

20. $\int_{b}^{\cdot \infty} E_{1}(a x) \frac{d x}{x^{2}}=\frac{1}{b}\left[(1+a b) E_{1}(a b)-e^{-a b}\right]$

21. $\int E_{1}(a x+b) \frac{d x}{x^{2}}=\frac{1}{b}\left[a e^{-b} E_{1}(a x)-\frac{1}{x}(a x+b) E_{1}(a x+b)\right]$

22. $\int E_{1}(a x) \frac{d x}{x^{n+2}}=-\frac{1}{(n+1)}\left[\frac{1}{x^{n+1}} E_{1}(a x)+\int e^{-a x} \frac{d x}{x^{n+2}}\right]$

23. $\int_{b}^{\infty} E_{1}(a x) \frac{d x}{x^{n+2}}=\frac{1}{(n+1)(n+1) ! b^{n+1}}\left[\left\{(n+1) !+(-1)^{n}(a b)^{n+1}\right\} E_{1}(a b)\right.$

$$
\left.-e^{-a b} \sum_{m=0}^{n}(n-m) !(-a b)^{m}\right]
$$

24. $\int E_{1}(a x+b) \frac{d x}{x^{n+2}}=-\frac{1}{(n+1)}\left\{\frac{1}{x^{n+1}}+(-1)^{n}\left(\frac{a}{b}\right)^{n+1}\right\} E_{1}(a x+b)$

$$
\begin{aligned}
& +\frac{(-1)^{n}}{(n+1)}\left(\frac{a}{b}\right)^{n+1} e_{n}(b) e^{-b} E_{1}(a x) \\
& +\frac{(-1)^{n}}{(n+1)}\left(\frac{a}{b}\right)^{n+1} e^{-(a x+b)} \sum_{m=1}^{n} \frac{1}{m !}\left(-\frac{b}{a x}\right)^{m} \sum_{k=0}^{m-1}(m-k-1) !(-a x)^{k}
\end{aligned}
$$

25. $\int E_{1}(a x) \frac{d x}{x^{p}}=-\frac{1}{(p-1)}\left[\frac{1}{x^{p-1}} E_{1}(a x)+\int e^{-a x} \frac{d x}{x^{p}}\right] \quad p>1$

26. $\int_{b}^{\infty} E_{1}(a x) \frac{d x}{x^{n+p+1}}=\frac{1}{(n+p)} \cdot \frac{1}{b^{n+p}} E_{1}(a b)+(-1)^{n} \frac{a^{n+p}}{(n+p)} \frac{\Gamma(p)}{\Gamma(n+p+1)} \Gamma(1-p, a b)$

$$
-\frac{1}{(n+p)} \cdot \frac{1}{b^{n+p}} \cdot \frac{e^{-a b}}{\Gamma(n+p+1)} \sum_{m=0}^{n}(-a b)^{m} \Gamma(n-m+p) \quad 0<p<1
$$

27. $\int_{0}^{\infty} E_{1}\left(a x^{2}\right) d x=\sqrt{\frac{\pi}{a}}$

28. $\int_{0}^{\infty} x^{p}(x+a)^{-p-2} E_{1}(b x) d x=\frac{1}{a} \cdot \frac{1}{\sqrt{a b}} \cdot \frac{\Gamma(p+1)}{(p+1)} e^{a b / 2} W_{-(p+1 / 2), 0}(a b) \quad p>-1$

29. $\int_{0}^{\infty}(x+a)^{-2} E_{1}(b x) d x=\frac{1}{a} e^{a b} E_{1}(a b)$ 
30. $\int_{0}^{\infty} x^{p}(x+a)^{-p}\left\{1+\frac{2 x}{a(p+1)}\right\} E_{1}(x) d x=\frac{1}{\sqrt{a}} \cdot \frac{\Gamma(p+1)}{(p+1)} e^{a / 2} W_{1 / 2-p,-1}(a) \quad p>-1$

31. $\int_{0}^{\infty} x^{p}(x+a)^{p}\left\{1+\frac{2 x}{a}\right\} E_{1}(x) d x=a^{p-1 / 2} \frac{\Gamma(p+1)}{(p+1)} e^{a / 2} W_{1 / 2,-p-1}(a) \quad p>-1$

32. $\int_{0}^{\infty} x^{p}(x+a)^{-p-2} E_{1}(x+a) d x=a^{-3 / 2} \Gamma(p+1) e^{-a / 2} W_{-(p+3 / 2), 0}(a) \quad p>-1$

\subsection{Combination of Exponential Integral With Exponentials and Powers}

1. $\int e^{-a x} E_{1}(b x) d x=\frac{1}{a}\left[E_{1}\{(a+b) x\}-e^{-a x} E_{1}(b x)\right]$

2. $\int e^{a x} E_{1}(b x) d x=-\frac{1}{a}\left[E_{1}\{(b-a) x\}-e^{a x} E_{1}(b x)\right] \quad b>a$

3. $\int_{0}^{\infty} e^{-a x} E_{1}(b x) d x=\frac{1}{a} \ln \left(1+\frac{a}{b}\right)$

4. $\int_{0}^{\infty} e^{a x} E_{1}(b x) d x=-\frac{1}{a} \ln \left(1-\frac{a}{b}\right)$

$b>a$

5. $\int_{0}^{c} e^{a x} E_{1}(a x) d x=\frac{1}{a}\left[\gamma+\ln (a c)+e^{a c} E_{1}(a c)\right]$

6. $\int_{0}^{c} e^{a x} E_{1}(b x) d x=-\frac{1}{a}\left[E_{1}\{(a-b) c\}-e^{a c} E_{1}(b c)+\ln \left(\frac{a}{b}-1\right)\right] \quad a>b$

7. $\int e^{-a x} \operatorname{Ei}(b x) d x=-\frac{1}{a}\left[E_{1}\{(a-b) x\}+e^{-a x} E i(b x)\right] \quad a>b$

8. $\int_{0}^{\infty} e^{-a x} \operatorname{Ei}(b x) d x=-\frac{1}{a} \ln \left(\frac{a}{b}-1\right) \quad a>b$

9. $\int_{0}^{c} e^{-a x} \operatorname{Ei}(a x) d x=\frac{1}{a}\left[\gamma+\ln (a c)-e^{-a c} \operatorname{Ei}(a c)\right]$

10. $\int x e^{-a x} E_{1}(b x) d x=\frac{1}{a^{2}}\left[E_{1}\{(a+b) x\}-(1+a x) e^{-a x} E_{1}(b x)+\left(\frac{a}{a+b}\right) e^{-(a+b) x}\right]$

11. $\int_{0}^{\infty} x e^{-a x} E_{1}(b x) d x=\frac{1}{a^{2}}\left[\ln \left(1+\frac{a}{b}\right)-\frac{a}{a+b}\right]$

12. $\int_{0}^{c} x e^{a x} E_{1}(a x) d x=\frac{1}{a^{2}}\left[a c-\gamma-\ln (a c)-(1-a c) e^{a c} E_{1}(a c)\right]$

13. $\int x e^{c x} E_{1}(a x+b) d x=\frac{1}{c}\left(x-\frac{1}{c}\right) e^{c x} E_{1}(a x+b)-\frac{1}{c(a-c)} e^{-\{(a-c) x+b\}}$

$$
+\frac{1}{a c^{2}}(a+b c) e^{-b c / a} E_{1}\left\{\frac{(a-c)(a x+b)}{a}\right\} \quad a>c
$$

14. $\int x e^{a x} E_{1}(a x+b) d x=\frac{1}{a}\left(x-\frac{1}{a}\right) e^{a x} E_{1}(a x+b)+\frac{1}{a}\left\{x-\frac{1}{a}(1+b) \ln (a x+b)\right\} e^{-b}$

15. $\int_{0}^{\infty} x e^{-a x} E i(b x) d x=-\frac{1}{a^{2}}\left[\ln \left(\frac{a}{b}-1\right)-\frac{a}{a-b}\right] \quad a>b$ 
16. $\int x^{n} e^{-a x} E_{1}(b x) d x=\frac{n !}{a^{n+1}} E_{1}\{(a+b) x\}-\frac{n !}{a^{n+1}} e_{n}(a x) e^{-a x} E_{1}(b x)$

$$
+\frac{n !}{a^{n+1}} e^{-(a+b) x} \sum_{m=1}^{n} \frac{e_{m-1}\{(a+b) x\}}{m\left(1+\frac{b}{a}\right)^{m}}
$$

17. $\int_{0}^{\infty} x^{n} e^{-a x} E_{1}(b x) d x=\frac{n !}{a^{n+1}}\left[\ln \left(1+\frac{a}{b}\right)-\sum_{m=1}^{n} \frac{1}{m}\left(\frac{a}{a+b}\right)^{m}\right]$

18. $\int_{0}^{\infty} x^{n} e^{-a x} \operatorname{Ei}(b x) d x=\frac{-n !}{a^{n+1}}\left[\ln \left(\frac{a}{b}-1\right)-\sum_{m=1}^{n} \frac{1}{m}\left(\frac{a}{a-b}\right)^{m}\right] \quad a>b$

19. $\int x^{p} e^{-a x} E_{1}(b x) d x=\frac{1}{a^{p+1}} \gamma(p+1, a x) E_{1}(b x)+\frac{1}{b^{p+1}} \sum_{m=0}^{\infty} \frac{\gamma(p+m+1, b x)}{m !(p+m+1)}\left(\frac{-a}{b}\right)^{m}$

20. $\int_{0}^{\infty} x^{p} e^{-a x} E_{1}(b x) d x=\frac{\Gamma(p+1)}{p+1} \cdot \frac{1}{(a+b)^{p+1}}{ }_{2} F_{1}\left(1, p+1 ; p+2 ; \frac{a}{a+b}\right)$

$$
\begin{aligned}
& =\frac{\Gamma(p+1)}{a^{p+1}} B_{a /(a+b)}(p+1,0) \\
& =\frac{\Gamma(p+1)}{(a+b)^{p+1}} \sum_{m=0}^{\infty} \frac{1}{p+m+1}\left(\frac{a}{a+b}\right)^{m} \quad p>-1
\end{aligned}
$$

21. $\int_{0}^{\infty} x^{p} e^{a x} E_{1}(b x) d x=\frac{\Gamma(p+1)}{(p+1)} \cdot \frac{1}{b^{p+1}}{ }_{2} F_{1}\left(p+1, p+1 ; p+2 ; \frac{a}{b}\right) \quad b>a, p>-1$

22. $\int_{0}^{\infty} x^{p} e^{a x} E_{1}(a x) d x=-\frac{\pi}{\sin (p \pi)} \frac{\Gamma(p+1)}{a^{p+1}}$

$$
-1<p<0
$$

23. $\int_{0}^{\infty} x^{p} e^{-a x} \operatorname{Ei}(a x) d x=-\pi \cot (p \pi) \frac{\Gamma(p+1)}{a^{p+1}}$

24. $\int_{0}^{\infty} x^{p-1} e^{x} E_{1}(x+a) d x=\Gamma(p) \Gamma(1-p) e^{-a / 2} a^{(p-1) / 2} W_{(p-1) / 2, p / 2}(a)$ $-1<p<0$

$25 . \int_{-\ln b}^{\infty} e^{-a x}\left\{E_{1}\left(e^{-x}\right)-E_{1}(b)\right\} d x=\frac{1}{a} \gamma(a, b)$ $b<1$

26. $\int_{0}^{\infty} x^{-1 / 2} e^{-a x} E_{1}(b x) d x=2 \sqrt{\frac{\pi}{a}} \ln \left(\sqrt{\frac{a}{b}}+\sqrt{\frac{a+b}{b}}\right)$

27. $\int_{0}^{\infty} x^{-1 / 2} e^{a x} E_{1}(b x) d x=2 \sqrt{\frac{\pi}{a}} \sin ^{-1}\left(\sqrt{\frac{a}{b}}\right)$ $b \geqslant a$

28. $\int e^{-a x} E_{1}(b x) \frac{d x}{x}=-\int e^{-b x} E_{1}(a x) \frac{d x}{x}-E_{1}(a x) E_{1}(b x)$

29. $\int_{c}^{\infty} e^{-a x} E_{1}(b x) \frac{d x}{x}=\left[\gamma+\ln a c+E_{1}(a c)\right] E_{1}(b c)+\frac{1}{2}\left[\zeta(2)+(\gamma+\ln b c)^{2}\right]$

$$
+e^{-b c} \sum_{m=0}^{\infty} \frac{e_{m}(b c)}{(m+1)^{2}}\left(-\frac{a}{b}\right)^{m+1}+\sum_{m=1}^{\infty} \frac{(-b c)^{m}}{m ! m^{2}}
$$

30. $\int e^{-a x} E_{1}(a x) \frac{d x}{x}=-\frac{1}{2}\left[E_{1}(a x)\right]^{2}$ 
31. $\int_{0}^{\infty}\left(1-e^{-a x}\right) E_{1}(b x) \frac{d x}{x}=\int_{0}^{a / b} \ln (1+x) \frac{d x}{x}$

$$
\begin{aligned}
& =-L_{2}\left(-\frac{a}{b}\right) \\
& =-\sum_{m=0}^{\infty} \frac{(-a / b)^{m+1}}{(m+1)^{2}}
\end{aligned}
$$

$$
b \geqslant a
$$

32. $\int_{0}^{\infty}\left[\frac{e^{a x} E_{1}(a x)}{x+b}-\frac{e^{-a x} E i(a x)}{x-b}\right] d x=\pi^{2} e^{-a b}$

$$
a>0
$$

$$
=0
$$

33. $\int_{0}^{a}(a-x)^{p-1} x^{-p} e^{x} E_{1}(x) d x=a^{-1 / 2} \Gamma(p) \Gamma(1-p) \Gamma(1-p) W_{p-1 / 2,0}(a) \quad 0<p<1$

34. $\int_{-\infty}^{\infty} e^{a x} e^{-i b x} E_{1}(a x) d x=\frac{\pi}{(b+i a)} \operatorname{sgn} b$

35. $\int_{-\infty}^{\infty} e^{-a x} e^{-i b x} \operatorname{Ei}(a x) d x=\frac{-\pi}{(b-i a)} \operatorname{sgn} b$

36. $\int_{0}^{\infty} e^{-a x^{2}} E_{1}\left(b x^{2}\right) d x=\sqrt{\frac{\pi}{a}} \ln \left(\sqrt{\frac{a}{b}}+\sqrt{\frac{a+b}{b}}\right)$

37. $\int_{0}^{\infty} \tilde{e}^{a x^{2}} E_{1}\left(b x^{2}\right) d x=\sqrt{\frac{\pi}{a}} \sin ^{-1}\left(\sqrt{\frac{a}{b}}\right) \quad b \geqslant a$

38. $\int_{0}^{\infty} e^{-a x} E_{1}\left(\frac{b}{x}\right) d x=\frac{2}{a} K_{0}(2 \sqrt{a b})$

39. $\int_{0}^{\infty} e^{-a x^{2}} E_{1}\left(\frac{b}{x^{2}}\right) d x=\sqrt{\frac{\pi}{a}} E_{1}(2 \sqrt{a b})$

40. $\int_{0}^{\infty} e^{-a x^{2}} E_{1}\left(\frac{b}{x^{2}}\right) \frac{d x}{x^{2}}=\sqrt{\frac{\pi}{b}} e^{-2 \sqrt{a b}}-2 \sqrt{a \pi} E_{1}(2 \sqrt{a b})$

41. $\int_{0}^{\infty} e^{-a^{2} x^{2}} e^{b^{2} / x^{2}} E_{1}\left(\frac{b^{2}}{x^{2}}\right) d x=-\frac{\sqrt{\pi}}{a}[\cos (2 a b) C i(2 a b)+\sin (2 a b) \operatorname{si}(2 a b)]$

42. $\int_{0}^{\infty} e^{-a^{2} x^{2}} e^{b^{2} / x^{2}} E_{1}\left(\frac{b^{2}}{x^{2}}\right) \frac{d x}{x^{2}}=-\frac{\sqrt{\pi}}{b}[\cos (2 a b) \operatorname{si}(2 a b)-\sin (2 a b) C i(2 a b)]$

43. $\int_{0}^{\infty} \cosh (a x) E_{1}(b x) d x=\frac{1}{2 a} \ln \left(\frac{b+a}{b-a}\right) \quad b>a$

44. $\int_{0}^{\infty} \sinh (a x) E_{1}(b x) \frac{d x}{x}=\sum_{m=0}^{\infty} \frac{(a / b)^{2 m+1}}{(2 m+1)^{2}}$

\subsection{Combination of Exponential Integral With Trigonometric Functions}

1. $\int_{0}^{\infty} E_{1}(a x) \sin (b x) d x=\frac{1}{2 b} \ln \left(1+\frac{b^{2}}{a^{2}}\right)$

2. $\int_{0}^{\infty} E_{1}(a x) \cos (b x) d x=\frac{1}{b} \tan ^{-1}\left(\frac{b}{a}\right)$ 
3. $\int_{0}^{\infty} E_{1}(a x) \frac{\sin (2 n x)}{\sin x} d x=2 \sum_{m=0}^{n-1} \frac{1}{(2 m+1)} \tan ^{-1}\left(\frac{2 m+1}{a}\right)$

4. $\int_{0}^{\infty} E_{1}(a x) \frac{\sin [(2 n+1) x]}{\sin x} d x=\frac{1}{a}+\sum_{m=1}^{n} \frac{1}{m} \tan ^{-1}\left(\frac{2 m}{a}\right)$

5. $\int_{0}^{\infty} \sin (a \sqrt{x}) E_{1}(x) d x=\frac{2 \pi}{a^{2}} \operatorname{erf}\left(\frac{a}{2}\right)-\frac{2}{a} \sqrt{\pi} e^{-a^{2 / 4}}$

6. $\int_{0}^{\infty} x^{-1 / 2} \cos (a \sqrt{x}) E_{1}(x) d x=\frac{2 \pi}{a} \operatorname{erf}\left(\frac{a}{2}\right)$

7. $\int_{0}^{\infty} x^{p-1 / 2} \sin (a \sqrt{x}) E_{1}(x) d x=a \frac{\Gamma(p+1)}{(p+1)}{ }_{2} F_{2}\left(p+1, p+1 ; \frac{3}{2}, p+2 ;-\frac{a^{2}}{4}\right) \quad p>-1$

8. $\int_{0}^{\infty} x^{p} \cos (a \sqrt{x}) E_{1}(x) d x=\frac{\Gamma(p+1)}{(p+1)}{ }_{2} F_{2}\left(p+1, p+1 ; \frac{1}{2}, p+2 ;-a^{2} / 4\right) \quad p>-1$

9. $\int_{0}^{\infty} E_{1}(a x) \sin b x e^{-c x} d x=\frac{1}{\left(b^{2}+c^{2}\right)}\left[\frac{b}{2} \ln \left\{\frac{(a+c)^{2}+b^{2}}{a^{2}}\right\}-c \tan ^{-1}\left(\frac{b}{a+c}\right)\right]$

10. $\int_{0}^{\infty} E_{1}(a x) \sin (b x) e^{c x} d x=\frac{1}{\left(b^{2}+c^{2}\right)}\left[\frac{b}{2} \ln \left\{\frac{(a-c)^{2}+b^{2}}{a^{2}}\right\}+c \tan ^{-1}\left(\frac{b}{a-c}\right)\right] \quad a \geqslant c$

11. $\int_{0}^{\infty} E i(a x) \sin (b x) e^{-c x} d x=\frac{1}{\left(b^{2}+c^{2}\right)}\left[-\frac{b}{2} \ln \left\{\frac{(c-a)^{2}+b^{2}}{a^{2}}\right\}+c \tan ^{-1}\left(\frac{b}{c-a}\right)\right] \quad c \geqslant a$

12. $\int_{0}^{\infty} E_{1}(a x) \cos (b x) e^{-c x} d x=\frac{1}{\left(b^{2}+c^{2}\right)}\left[\frac{c}{2} \ln \left\{\frac{(a+c)^{2}+b^{2}}{a^{2}}\right\}+b \tan ^{-1}\left(\frac{b}{a+c}\right)\right]$

13. $\int_{0}^{\infty} E_{1}(a x) \cos (b x) e^{c x} d x=\frac{1}{\left(b^{2}+c^{2}\right)}\left[-\frac{c}{2} \ln \left\{\frac{(a-c)^{2}+b^{2}}{a^{2}}\right\}+b \tan ^{-1}\left(\frac{b}{c-a}\right)\right] \quad a \geqslant c$

14. $\int_{0}^{\infty} E i(a x) \cos (b x) e^{-c x} d x=\frac{1}{\left(b^{2}+c^{2}\right)}\left[-\frac{c}{2} \ln \left\{\frac{(c-a)^{2}+b^{2}}{a^{2}}\right\}-b \tan ^{-1}\left(\frac{b}{c-a}\right)\right] \quad c \geqslant a$

15. $\int_{0}^{\infty} E_{1}(a x) \sin ^{2}\left(\frac{1}{2} b x\right) e^{-c x} d x=\frac{1}{2 c} \ln \left(1+\frac{c}{a}\right)-\frac{1}{2} \cdot \frac{1}{\left(b^{2}+c^{2}\right)}$

$$
\times\left[\frac{c}{2} \ln \left\{\frac{(a+c)^{2}+b^{2}}{a^{2}}\right\}+b \tan ^{-1}\left(\frac{b}{a+c}\right)\right]
$$

16. $\int_{0}^{\infty} E_{1}(a x) \cos ^{2}\left(\frac{1}{2} b x\right) e^{-c x} d x=\frac{1}{2 c} \ln \left(1+\frac{c}{a}\right)+\frac{1}{2} \cdot \frac{1}{\left(b^{2}+c^{2}\right)}$

$$
\times\left[\frac{c}{2} \ln \left\{\frac{(a+c)^{2}+b^{2}}{a^{2}}\right\}+b \tan ^{-1}\left(\frac{b}{a+c}\right)\right] .
$$

\subsection{Combination of Exponential Integral With Logarithms and Powers}

1. $\int \ln x E_{1}(b x) d x=\frac{1}{b}\left[(1-\ln x) e^{-b x}-(1+b x-b x \ln x) E_{1}(b x)\right]$

2. $\int_{0}^{\infty} \ln x E_{1}(b x) d x=-\frac{1}{b}(1+\gamma+\ln b)$

3. $\int \ln x E i(b x) d x=\frac{1}{b}\left[(1-\ln x) e^{b x}+(1-b x+b x \ln x) E i(b x)\right]$ 
4. $\int x \ln x E_{1}(b x) d x=\frac{1}{2 b^{2}}\left\{\frac{1}{2}(1+b x)-(1+b x) \ln x-1\right\} e^{-b x}$

$$
-\frac{1}{2 b^{2}}\left(1+\frac{1}{2} b^{2} x^{2}-b^{2} x^{2} \ln x\right) E_{1}(b x)
$$

5. $\int_{0}^{\infty} x \ln x E_{1}(b x) d x=-\frac{1}{2 b^{2}}\left(-\frac{1}{2}+\gamma+\ln b\right)$

6. $\int x^{n} \ln x E_{1}(b x) d x=\frac{n !}{(n+1) b^{n+1}}\left[e_{n}(b x)\left(\frac{1}{n+1}-\ln x\right)-\sum_{m=0}^{n-1} \frac{e_{m}(b x)}{(m+1)}\right] e^{-b x}$

$$
-\frac{n !}{(n+1) b^{n+1}}\left[1+\frac{(b x)^{n+1}}{(n+1) !}\{1-(n+1) \ln x\}\right] E_{1}(b x)
$$

7. $\int_{0}^{\infty} x^{n} \ln x E_{1}(b x) d x=\frac{-n !}{(n+1) b^{n+1}}\left[\gamma+\ln b+\frac{1}{n+1}-\sum_{m=1}^{n} \frac{1}{m}\right]$

8. $\int x^{p} \ln x E_{1}(b x) d x=\frac{1}{(p+1) b^{p+1}}\left\{\ln x-\frac{1}{(p+1)}\right\}\left[\gamma(p+1, b x)+(b x)^{p+1} E_{1}(b x)\right]$

$$
-\frac{x^{p+1}}{p+1} \sum_{m=0}^{\infty} \frac{(-b x)^{m}}{m !(p+m+1)^{2}} \quad p>-1
$$

9. $\int_{0}^{\infty} x^{p} \ln x E_{1}(b x) d x=\frac{-\Gamma(p+1)}{(p+1)} \cdot \frac{1}{b^{p+1}}\left[\ln b+\frac{1}{p+1}-\Psi(p+1)\right] \quad p>-1$.

\subsection{Combination of Exponential Integral With Logarithms, Exponentials, and Powers}

1. $\int e^{-a x} \ln x E_{1}(b x) d x=-\frac{1}{a} \int e^{-b x} E_{1}(a x) \frac{d x}{x}+\frac{\ln x}{a}\left[\gamma+\ln \{(a+b) x\}+E_{1}\{(a+b) x\}\right]$

$$
-\frac{1}{a}\left[\ln x e^{-a x}+E_{1}(a x)\right] E_{1}(b x)-\frac{1}{2 a} \ln ^{2} x+\frac{1}{a} \sum_{m=1}^{\infty} \frac{\{-(a+b) x\}^{m}}{m ! m^{2}}
$$

2. $\int_{0}^{\infty} e^{-a x} \ln x E_{1}(b x) d x=-\frac{1}{a}\left[\ln \left(1+\frac{a}{b}\right)\{\gamma+\ln (a+b)\}+\left(\frac{a}{a+b}\right) \Phi\left(\frac{a}{a+b}, 2,1\right)\right]$

3. $\int_{0}^{\infty} e^{-a x} \ln x E_{1}(a x) d x=-\frac{1}{2 a}\left[\zeta(2)+(\gamma+\ln a) \ln 4+\ln ^{2} 2\right]$

4. $\int_{0}^{\infty} x e^{-a x} \ln x E_{1}(b x) d x=-\frac{1}{a^{2}}\left[\left\{\ln \left(1+\frac{a}{b}\right)-\frac{a}{a+b}\right\}(\gamma+\ln (a+b)-1)\right.$

$$
\left.+\left(\frac{a}{a+b}\right)^{2} \Phi\left(\frac{a}{a+b}, 2,2\right)\right]
$$

5. $\int_{0}^{\infty} x^{n} e^{-a x} \ln x E_{1}(b x) d x=-\frac{n !}{a^{n+1}}\left\{\ln \left(1+\frac{a}{b}\right)-\sum_{m=1}^{n} \frac{1}{m}\left(\frac{a}{a+b}\right)^{m}\right\}\left[\gamma+\ln (a+b)-\sum_{m=1}^{n} \frac{1}{m}\right]$

$$
-\frac{n !}{(a+b)^{n+1}} \Phi\left(\frac{a}{a+b}, 2, n+1\right)
$$

6. $\int_{0}^{\infty} x^{p} e^{-a x} \ln x E_{1}(b x) d x=\frac{\Gamma(p+1)}{(a+b)^{p+1}}\left[\{\Psi(p+1)-\ln (a+b)\} \Phi\left(\frac{a}{a+b}, 1, p+1\right)\right.$

$$
\left.-\Phi\left(\frac{a}{a+b}, 2, p+1\right)\right]
$$




\subsection{Combination of Two Exponential Integrals}

1. $\int E_{1}(a x) E_{1}(b x) d x=x E_{1}(a x) E_{1}(b x)+\left(\frac{1}{a}+\frac{1}{b}\right) E_{1}\{(a+b) x\}$

$$
-\frac{1}{a} e^{-a x} E_{1}(b x)-\frac{1}{b} e^{-b x} E_{1}(a x)
$$

2. $\int_{0}^{\infty} E_{1}(a x) E_{1}(b x) d x=\left(\frac{1}{a}+\frac{1}{b}\right) \ln (a+b)-\frac{1}{a} \ln b-\frac{1}{b} \ln a$

3. $\int E_{1}(a x) E_{i}(b x) d x=x E_{1}(a x) E_{i}(b x)+\left(\frac{1}{b}-\frac{1}{a}\right) E_{1}\{(a-b) x\}$

$$
-\frac{1}{a} e^{-a x} E i(b x)-\frac{1}{b} e^{b x} E_{1}(a x)
$$

4. $\int_{0}^{\infty} E_{1}(a x) E i(b x) d x=\frac{1}{a}\left[\left(\frac{a-b}{b}\right) \ln \left(\frac{a-b}{b}\right)-\left(\frac{a}{b}\right) \ln \left(\frac{a}{b}\right)\right] \quad a>b$

5. $\int E_{1}(a x) E i(a x) d x=x E_{1}(a x) E i(a x)-\frac{1}{a}\left[e^{-a x} E i(a x)+e^{a x} E_{1}(a x)\right]$

6. $\int_{0}^{\infty} E_{1}(a x) E i(a x) d x=0$

7. $\int x E_{1}(a x) E_{1}(b x) d x=\frac{x^{2}}{2} E_{1}(a x) E_{1}(b x)+\frac{1}{2}\left(\frac{1}{a^{2}}+\frac{1}{b^{2}}\right) E_{1}\{(a+b) x\}$

$$
\begin{aligned}
& -\frac{1}{2 a^{2}} e_{1}(a x) e^{-a x} E_{1}(b x)-\frac{1}{2 b^{2}} e_{1}(b x) e^{-b x} E_{1}(a x) \\
& +\frac{1}{2 a b} e^{-(a+b) x}
\end{aligned}
$$

8. $\int_{0}^{\infty} x E_{1}(a x) E_{1}(b x) d x=\frac{1}{2}\left(\frac{1}{a^{2}}+\frac{1}{b^{2}}\right) \ln (a+b)-\frac{1}{2 a^{2}} \ln b-\frac{1}{2 b^{2}} \ln a-\frac{1}{2 a b}$

9. $\int x^{n} E_{1}(a x) E_{1}(b x) d x=\frac{x^{n+1}}{(n+1)} E_{1}(a x) E_{1}(b x)+\frac{n !}{(n+1)}\left(\frac{1}{a^{n+1}}+\frac{1}{b^{n+1}}\right) E_{1}\{(a+b) x\}$

$$
\begin{aligned}
& -\frac{n !}{a^{n+1}} \frac{1}{(n+1)} e_{n}(a x) e^{-a x} E_{1}(b x) \\
& -\frac{n !}{b^{n+1}}: \frac{1}{(n+1)} e_{n}(b x) e^{-b x} E_{1}(a x) \\
& +\frac{n !}{(n+1)} e^{-(a+b) x} \sum_{m=1}^{n} \frac{e_{m-1}\{(a+b) x\}}{m(a+b)^{m}}\left[\frac{a^{m}}{a^{n+1}}+\frac{b^{m}}{b^{n+1}}\right]
\end{aligned}
$$

10. $\int_{0}^{\infty} x^{n} E_{1}(a x) E_{1}(b x) d x=-\frac{n !}{(n+1)}\left[\frac{1}{a^{n+1}}\left\{\ln \left(\frac{b}{a+b}\right)+\sum_{m=1}^{n} \frac{1}{m}\left(\frac{a}{a+b}\right)^{m}\right\}\right.$

$$
\left.+\frac{1}{b^{n+1}}\left\{\ln \left(\frac{a}{a+b}\right)+\sum_{m=1}^{n} \frac{1}{m}\left(\frac{b}{a+b}\right)^{m}\right\}\right]
$$

11. $\int_{0}^{\infty} x^{p}\left[E_{1}(x)\right]^{2} d x=2^{-p} \frac{\Gamma(p+1)}{(p+1)} \sum_{m=0}^{\infty} \frac{(1 / 2)^{m}}{(m+p+1)}$

$$
=2^{-p} \frac{\Gamma(p+1)}{(p+1)} \Phi\left(\frac{1}{2}, 1, p+1\right) \quad p>-1
$$


12. $\int_{0}^{a} E_{1}(x) E_{1}(a-x) d x=2(\gamma+\ln a) e^{-a}+2(1-a \gamma-a \ln a) E_{1}(a)$

$$
-a\left\{\zeta(2)+(\gamma+\ln a)^{2}\right\}-2 a \sum_{m=1}^{\infty} \frac{(-a)^{m}}{m ! m^{2}}
$$

13. $\int_{a}^{\infty} E_{1}(x) E_{1}(x-a) d x=e^{-a}\left\{\ln 2-e^{2 a} E_{1}(2 a)\right\}+\frac{1}{2} a\left\{[\gamma+\ln a]^{2}-2 \zeta(2)\right\}$

$$
\begin{aligned}
& -a(\gamma+\ln a) E i(a)-a \ln 2\left\{E_{1}(a)+E i(a)\right\} \\
& +E_{1}(a)+a \sum_{m=1}^{\infty} b_{m}\left(\frac{a^{m}}{m \cdot m !}\right)
\end{aligned}
$$

where

$$
b_{2 m}=\frac{1}{2 m}+2 \sum_{n=1}^{m} \frac{1}{(2 n-1)} \text {, }
$$

and

$$
b_{2 m+1}=\frac{1}{(2 m+1)}+2 \sum_{n=1}^{m+1} \frac{1}{(2 n-1)}
$$

14. $\int_{0}^{\infty} x^{-1 / 2} E_{1}\left(\frac{x}{a^{2}}\right) E_{1}\left(\frac{a^{2} b^{2}}{4 x}\right) d x=4 a \sqrt{\pi}\left[(1+b) E_{1}(b)-e^{-b}\right]$

15. $\int_{0}^{\infty} E_{1}\left(\frac{x^{2}}{a^{2}}\right) E_{1}\left(\frac{a^{2} b^{2}}{4 x^{2}}\right) d x=2 a \sqrt{\pi}\left[(1+b) E_{1}(b)-e^{-b}\right]$

16. $c \int_{0}^{\infty} E_{1}(a x) E_{1}(b x) e^{-c x} d x=\frac{\pi^{2}}{6}-L_{2}\left(\frac{a}{a+b+c}\right)-L_{2}\left(\frac{b}{a+b+c}\right)+\ln a \ln b$

$$
\begin{aligned}
& +\ln (a+c) \ln \left(\frac{a+b+c}{b}\right)+\ln (b+c) \ln \left(\frac{a+b+c}{a}\right) \\
& -\ln ^{2}(a+b+c)
\end{aligned}
$$

17. $\int_{0}^{\infty} e^{-x} E_{1}(x) E_{1}(x) d x=\frac{\pi^{2}}{6}-2 L_{2}\left(\frac{1}{3}\right)+2 \ln 2 \ln 3-\ln ^{2} 3$

$$
=1.228558 \ldots \text {. }
$$

18. $\int_{0}^{\infty} e^{x} E_{1}(x) E_{1}(x) d x=\frac{\pi^{2}}{6}$

19. $\int_{0}^{\infty} e^{2 x} E_{1}(x) E_{1}(x) d x=\frac{\pi^{2}}{12}$

20. $a \int_{0}^{\infty} e^{a x} E_{1}(x) E_{1}\{(a+1) x\} d x=\frac{\pi^{2}}{12}+\frac{1}{2} \ln ^{2} 2-L_{2}\left(\frac{1-a}{2}\right)+\ln 2 \ln \left(\frac{1+a}{2}\right)$

$$
\begin{aligned}
= & \frac{\pi^{2}}{12}+\frac{1}{2} \ln ^{2}(1+a) \\
& +\sum_{m=1}^{\infty} \frac{(-1)^{m}}{m^{2}}\left(\frac{1-a}{1+a}\right)^{m}
\end{aligned}
$$

21. $a \int_{0}^{\infty} e^{2 a x} E_{1}\{(a+1) x\} E_{1}\{(a+1) x\} d x=\frac{\pi^{2}}{12}+\sum_{m=1}^{\infty} \frac{(-1)^{m}}{m^{2}}\left(\frac{1-a}{1+a}\right)^{m}$

22. $\int_{0}^{\infty} e^{-x} E_{1}(x) E i(x) d x=-\frac{\pi^{2}}{12}$ 
23. $a \int_{0}^{\infty} e^{-a x} E_{1}(x) E i\{(a-1) x\} d x=-\frac{\pi^{2}}{12}-\frac{1}{2} \ln ^{2}(a-1)+\ln (a+1) \ln (a-1)$

$$
-\sum_{m=1}^{\infty} \frac{(-1)^{m}}{m^{2}}\left(\frac{1-a}{1+a}\right)^{m}
$$

24. $\int_{0}^{\infty} e^{-2 x} \operatorname{Ei}(x) E i(x) d x=\frac{\pi^{2}}{4}$

25. $c^{2} \int_{0}^{\infty} x E_{1}(a x) E_{1}(b x) e^{-c x} d x=\frac{\pi^{2}}{6}-L_{2}\left(\frac{a}{a+b+c}\right)-L_{2}\left(\frac{b}{a+b+c}\right)+\ln a \ln b$

$$
\begin{aligned}
& -\ln ^{2}(a+b+c)+\left[\ln (a+c)-\frac{c}{a+c}\right] \ln \left(\frac{a+b+c}{b}\right) \\
& +\left[\ln (b+c)-\frac{c}{b+c}\right] \ln \left(\frac{a+b+c}{a}\right)
\end{aligned}
$$

26. $\int_{0}^{\infty} x e^{-x} E_{1}(x) E_{1}(x) d x=\frac{\pi^{2}}{6}-2 L_{2}\left(\frac{1}{3}\right)+2 \ln 2 \ln 3-\ln ^{2} 3-\ln 3$

$$
=0.129946 \ldots \text {. }
$$

27. $\frac{c^{n+1}}{n !} \int_{0}^{\infty} x^{n} E_{1}(a x) E_{1}(b x) e^{-c x} d x=\frac{\pi^{2}}{6}-L_{2}\left(\frac{a}{a+b+c}\right)-L_{2}\left(\frac{b}{a+b+c}\right)$

$+\ln a \ln b-\ln ^{2}(a+b+c)$

$$
\begin{aligned}
& +\left\{\ln (a+c)-\sum_{m=1}^{n} \frac{1}{m}\left(\frac{c}{a+c}\right)^{m}\right\} \ln \left(\frac{a+b+c}{b}\right) \\
& +\left\{\ln (b+c)-\sum_{m=1}^{n} \frac{1}{m}\left(\frac{c}{b+c}\right)^{m}\right\} \ln \left(\frac{a+b+c}{a}\right) \\
& +\sum_{m=2}^{n} \frac{1}{m}\left[\sum _ { k = 1 } ^ { m - 1 } \frac { 1 } { ( m - k ) } \left\{\left(\frac{a+b+c}{a+c}\right)^{k}\right.\right. \\
& \left.\left.+\left(\frac{a+b+c}{b+c}\right)^{k}\right\}\right]\left(\frac{c}{a+b+c}\right)^{m}
\end{aligned}
$$

28. $\int_{0}^{\infty} x^{-1 / 2} e^{x / a^{2}} e^{a^{2} b^{2} /(4 x)} E_{1}\left(\frac{x}{a^{2}}\right) E_{1}\left(\frac{a^{2} b^{2}}{4 x}\right) d x=2 a \pi^{3 / 2} e^{b} E_{1}(b)$

29. $\int_{0}^{\infty} e^{x^{2 / a} a^{2}} e^{a^{2} b^{2} /\left(4 x^{2}\right)} E_{1}\left(\frac{x^{2}}{a^{2}}\right) E_{1}\left(\frac{a^{2} b^{2}}{4 x^{2}}\right) d x=a \pi^{3 / 2} e^{b} E_{1}(b)$

30. $\int_{0}^{\infty} E_{1}(x) d x \int_{0}^{\infty} E_{1}(y) e^{-a|x-y|} d y=\frac{4}{a} \ln 2-\frac{\pi^{2}}{6 a^{2}}-\frac{1}{a^{2}} \ln ^{2}(a+1)$

$$
+\frac{2}{a^{2}} \sum_{m=1}^{\infty} \frac{(-1)^{m+1}}{m^{2}}\left(\frac{1-a}{1+a}\right)^{m}
$$

31. $\int_{0}^{\infty} \ln x\left[E_{1}(x)\right]^{2} d x=-\left[\zeta(2)+2(\gamma+1) \ln 2+\ln ^{2} 2\right]$

32. $\int_{0}^{\infty} x \ln x E_{1}(x) E_{1}(x) d x=-\frac{1}{2}\left[\zeta(2)+\ln ^{2} 2-\gamma-\frac{1}{2}+2(\gamma-1) \ln 2\right]$

33. $\int_{0}^{\infty} x^{n} \ln x E_{1}(x) E_{1}(x) d x=-\frac{n !}{(n+1)}\left[\zeta(2)+\ln ^{2} 2-2 \gamma \sum_{m=1}^{n} \frac{1}{2^{m} m}+2(\gamma+A) \ln 2-2 B\right]$ 
where

$$
\begin{aligned}
& A=\frac{1}{n+1}-\sum_{m=1}^{n} \frac{1}{m}\left(1+\frac{1}{2^{m}}\right), \\
& B=\sum_{m=1}^{n} \frac{1}{2^{m} m}\left[\frac{1}{n+1}+\frac{1}{m}-\sum_{k=1}^{n} \frac{1}{k}\right]
\end{aligned}
$$

34. $\int_{0}^{\infty} x^{p} \ln x E_{1}(x) E_{1}(x) d x=\frac{\Gamma(p+1)}{(p+1)} 2^{p}\left[\left\{\Psi(p+1)-\frac{1}{p+1}-\ln 2\right\} \sum_{m=0}^{\infty} \frac{1}{2^{m}(m+p+1)}\right.$

$$
\left.-\sum_{m=0}^{\infty} \frac{1}{2^{m}(m+p+1)^{2}}\right] \quad p>-1
$$

35. $\int_{0}^{\infty} E_{1}(x) d x \int_{0}^{\infty} E_{1}(y) E_{1}(|x-y|) d y=4 \ln 2-\frac{\pi^{2}}{6}$

36. $\int_{0}^{\infty} E_{1}(x) d x \int_{0}^{x} E_{1}(y) E_{1}(x-y) d y=\int_{0}^{\infty} E_{1}(x) d x \int_{x}^{\infty} E_{1}(y) E_{1}(y-x) d y=2 \ln 2-\frac{\pi^{2}}{12}$

\subsection{Combination of Exponential Integral With Bessel Functions}

1. $\int_{0}^{\infty} E_{1}(a x) J_{0}(b x) d x=\frac{1}{b} \ln \left[\frac{b+\left(a^{2}+b^{2}\right)^{1 / 2}}{a}\right]$

$2 . \int_{0}^{\infty} x E_{1}(a x) J_{0}(b x) d x=\frac{1}{b^{2}}\left[1-a\left(a^{2}+b^{2}\right)^{-1 / 2}\right]$

3. $\int_{0}^{\infty} x^{p} E_{1}(a x) J_{p-1}(b x) d x=\frac{1}{a b}\left(\frac{b}{2}\right)^{p}\left(a^{2}+b^{2}\right)^{1 / 2-p} \frac{\Gamma(2 p)}{\Gamma(p+1)} \times{ }_{2} F_{1}\left(\frac{1}{2}, 1 ; p+1 ; \frac{-b^{2}}{a^{2}}\right)$

$$
=\frac{1}{b}\left(\frac{b}{2}\right)^{p}\left(a^{2}+b^{2}\right)^{-p} \frac{\Gamma(2 p)}{\Gamma(p+1)} \times{ }_{2} F_{1}\left(\frac{1}{2}, p ; p+1 ; \frac{b^{2}}{a^{2}+b^{2}}\right) \quad p>0
$$

4. $\int_{0}^{\infty} x^{2 q+1-p} E_{1}(a x) J_{p}(b x) d x=\frac{1}{2} \frac{(b / 2)^{p}}{a^{2 q+2}} \times \frac{\Gamma(2 q+2)}{\Gamma(p+1)} \times \frac{1}{(q+1)}$

$$
\times{ }_{3} F_{2}\left(q+1, q+1, q+\frac{3}{2} ; q+2, p+1 ; \frac{-b^{2}}{a^{2}}\right) \quad p, q>-1
$$

$5 . \int_{0}^{\infty} E_{1}(a / x) J_{1}(b x) d x=\frac{2}{b} K_{0}(\sqrt{2 a b}) J_{0}(\sqrt{2 a b})$

6. $\int_{0}^{\infty} E_{1}(a / x) Y_{1}(b x) d x=\frac{2}{b} K_{0}(\sqrt{2 a b}) Y_{0}(\sqrt{2 a b})$

7. $\int_{0}^{\infty} E_{1}(x) I_{0}(b x) d x=\frac{1}{b}\left(\frac{\pi}{2}-\cos ^{-1} b\right) \quad 0<b \leqslant 1$

8. $\int_{0}^{\infty} E_{1}(a / x) K_{1}(b x) d x=\frac{2}{b} K_{0}\left(e^{i \pi / 4} \sqrt{2 a b}\right) K_{0}\left(e^{-i \pi / 4} \sqrt{2 a b}\right)$

9. $\int_{0}^{\infty} x E_{1}(a x) I_{0}(b x) d x=-\frac{1}{b^{2}}\left[1-a\left(a^{2}-b^{2}\right)^{-1 / 2}\right] \quad a>b$

10. $\int_{0}^{\infty} E_{1}(a x) J_{0}(b \sqrt{x}) d x=\frac{4}{b^{2}}\left[1-e^{-b^{2} /(4 a)}\right]$ 
11. $\int_{0}^{\infty} x^{1 / 2} E_{1}(a x) J_{1}(b \sqrt{x}) d x=\frac{8}{b^{3}}\left[1-\left(1+\frac{b^{2}}{4 a}\right) e^{-b^{2} /(4 a)}\right]$

12. $\int_{0}^{\infty} x^{p / 2} E_{1}(a x) J_{p}(b \sqrt{x}) d x=\left(\frac{2}{b}\right)^{p+2} \gamma\left(p+1, \frac{b^{2}}{4 a}\right) \quad p>-1$

13. $\int_{0}^{\infty} x^{-1 / 2} E_{1}(a x) J_{1}(b \sqrt{x}) d x=\frac{2}{b}\left[\gamma+\ln \left(\frac{b^{2}}{4 a}\right)+E_{1}\left(\frac{b^{2}}{4 a}\right)\right]$

14. $\int_{0}^{\infty} x^{q-p / 2} E_{1}(a x) J_{p}(b \sqrt{x}) d x=\left(\frac{b}{2}\right)^{p} \frac{\Gamma(q+1)}{\Gamma(p+1)} \times \frac{1}{a^{q+1}(q+1)}$

$$
\times{ }_{2} F_{2}\left(q+1, q+1 ; p+1, q+2 ;-\frac{b^{2}}{4 a}\right) \quad p, q>-1
$$

15. $\int_{0}^{\infty} E_{1}(a x) Y_{0 .}(b \sqrt{x}) d x=\frac{\pi}{4 b^{2}}\left[\gamma+\ln \left(\frac{b^{2}}{4 a}\right)-e^{-b^{2} /(4 a)} E i\left(\frac{b^{2}}{4 a}\right)\right]$

16. $\int_{0}^{\infty} E_{1}(a x) I_{0}(b \sqrt{x}) d x=\frac{4}{b^{2}}\left[e^{b^{2} /(4 a)}-1\right]$

17. $\int_{0}^{\infty} x^{n / 2} E_{1}(a x) I_{n}(b \sqrt{x}) d x=(-1)^{n+1} n !\left(\frac{2}{b}\right)^{n+2}\left[1-e_{n}\left(-\frac{b^{2}}{4 a}\right) e^{b^{2} /(4 a)}\right]$

18. $\int_{0}^{\infty} x^{q-(p+1) / 2} E_{1}(a x) \mathbf{H}_{p}(b \sqrt{x}) d x=\frac{2}{a} \sqrt{\frac{2}{\pi}} \frac{b^{p+1}}{(4 a)^{q}} \times \frac{\Gamma(q+1) \Gamma(q+1)}{\Gamma\left(p+\frac{3}{2}\right) \Gamma(q-p+2)}$

$$
\times_{3} F_{3}\left(1, q+1, q+1 ; \frac{3}{2}, p+\frac{3}{2}, q+2 ; \frac{-b^{2}}{4 a}\right)
$$

$$
q>-1, p>-\frac{3}{2}
$$

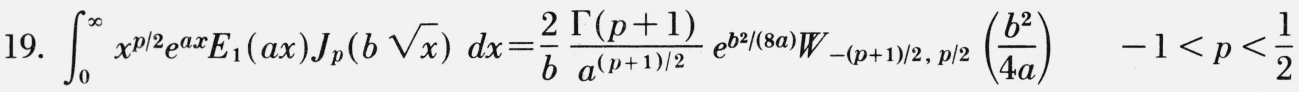

20. $\int_{0}^{\infty} x^{(p+1) / 2} e^{x} E_{1}(x) Y_{p}(b \sqrt{x}) d x=\frac{\sqrt{\pi}}{b} \Gamma\left(p+\frac{3}{2}\right) e^{b^{2} / 8} \times W_{-(p+2) / 2, p / 2}\left(b^{2} / 4\right)$

$$
-\frac{3}{2}<p<\frac{1}{2}
$$

21. $\int_{0}^{\infty} x^{(p+3) / 2} e^{x} E_{1}(x) Y_{p}(b \sqrt{x}) d x=\frac{-3 \sqrt{\pi}}{2 b} \Gamma\left(p+\frac{5}{2}\right) e^{b 2 / 8} \times W_{-(p+4) / 2, p / 2}\left(b^{2} / 4\right)$

$$
-\frac{5}{2}<p<-\frac{3}{2}
$$

22. $\int_{0}^{\infty} e^{a x} E_{1}(a x) \mathbf{H}_{0}(b \sqrt{x}) d x=\frac{\pi}{a} e^{b^{2} /(4 a)} \operatorname{erfc}\left(\frac{b}{2 \sqrt{a}}\right)$

23. $\int_{0}^{\infty} x^{-1 / 2} e^{x(1-a / 2)} K_{0}\left(\frac{1}{2} a x\right) E_{1}(x) d x=\left(\frac{\pi^{5}}{a}\right)^{1 / 2}{ }_{2} F_{1}\left(\frac{1}{2}, \frac{1}{2} ; 1 ; 1-\frac{1}{a}\right)$

24. $\int_{0}^{\infty} e^{-b x / 2} J_{0}(b x / 2) E_{1}(a x) d x=\frac{\sqrt{2}}{b} \ln \left[\frac{(a+b)+\sqrt{(a+b)^{2}+a^{2}}}{a(1+\sqrt{2})}\right]$

206 
$25 . \int_{0}^{\infty} e^{ \pm b x / 2} I_{0}\left(\frac{b x}{2}\right) E_{1}(a x) d x=\frac{2}{\sqrt{a}(\sqrt{a}+\sqrt{a \mp b)}} \quad a>b$ for upper sign

26. $\int_{0}^{\infty} x^{p} e^{x / 2} I_{p}\left(\frac{x}{2}\right) E_{1}(x) d x=\frac{\pi^{-1 / 2}}{p+\frac{1}{2}} \Gamma(1+2 p) \Gamma\left(\frac{1}{2}-p\right) \quad-\frac{1}{2}<p<\frac{1}{2}$

27. $\int_{0}^{\infty} x^{p} e^{(a-1) x} \boldsymbol{I}_{p}(x) E_{1}(a x) d x=\frac{\pi^{-1 / 2}}{a}\left(\frac{2}{a^{2}}\right)^{p} \frac{\Gamma(1+2 p) \Gamma\left(\frac{1}{2}-p\right)}{p+\frac{1}{2}}$

$$
\times{ }_{2} F_{1}\left(p+\frac{1}{2}, 2 p+1 ; p+\frac{3}{2} ; 1-\frac{2}{a}\right) \quad a \geqslant 1,-\frac{1}{2}<p<\frac{1}{2}
$$

28. $\int_{0}^{\infty} x E_{1}\left[a\left\{b+\left(b^{2}+x^{2}\right)^{1 / 2}\right\}\right] J_{0}(c x) d x=\frac{\exp \left\{-b\left(a+\sqrt{a^{2}+c^{2}}\right)\right\}}{\left(a+\sqrt{a^{2}+c^{2}}\right) \sqrt{a^{2}+c^{2}}}$

29. $\int_{0}^{\infty} E_{1}(a x) J_{0}(b \sqrt{x}) \ln x d x=\frac{4}{b^{2}} e^{-b^{2} /(4 a)}\left\{E i\left(\frac{b^{2}}{4 a}\right)+\ln a-\ln \left(\frac{b^{2}}{4 a}\right)\right\}$

$$
-\frac{8}{b^{2}}\left\{E_{1}\left(\frac{b^{2}}{4 a}\right)+\ln a+\ln \left(\frac{b^{2}}{4 a}\right)\right\}+\frac{4}{b^{2}}(\ln a-3 \gamma)
$$

30. $\int_{0}^{\infty} x^{p} E_{1}(x) J_{\lambda+\nu}(a \sqrt{x}) J_{\lambda-\nu}(a \sqrt{x}) d x=\frac{(a / 2)^{2 \lambda} \Gamma(p+\lambda+1)}{\Gamma(\lambda+\nu+1) \Gamma(\lambda-\nu+1)(p+\lambda+1)}$

$$
\begin{aligned}
& \times{ }_{4} F_{4}\left(\lambda+\frac{1}{2}, \lambda+1, \lambda+p+1,\right. \\
& \lambda+p+1 ; \lambda-\nu+1, \lambda+\nu+1, \\
&\left.2 \lambda+1, \lambda+p+2 ;-a^{2}\right) \quad \lambda+p+1>0
\end{aligned}
$$

31. $\int_{0}^{\infty} E_{1}(x / a)$ her $(2 \sqrt{x}) d x=\sin a$

32. $\int_{0}^{\infty} E_{1}(x / a)$ bee $(2 \sqrt{x}) d x=(1-\cos a)$

4.8. Combination of Exponential Integral With Other Special Functions

1. $\int_{0}^{\infty} E_{1}(a x) \gamma(p+1, b x) d x=\frac{1}{a} \frac{\Gamma(p+1)}{\left(1+\frac{a}{b}\right)^{p+1}}-\frac{1}{b} \int_{0}^{\infty} e^{-t} t^{(p+1)} E_{1}\left(\frac{a}{b} t\right) d t \quad p>-1$

See 4.2.20 for Evaluation of this integral.

2. $\int_{0}^{\infty} E_{1}(a x) \Gamma(p+1, b x) d x=\frac{1}{a} \Gamma(p+1)\left[1-\left(1+\frac{a}{b}\right)^{-p-1}\right]$

$$
+\frac{1}{b} \int_{0}^{\infty} e^{-t} t^{p+1} E_{1}(a t / b) d t \quad p>-1
$$

See 4.2.20 for Evaluation of this integral.

$350-890$ O-69-2

207 
3. $\int_{0}^{\infty} E_{1}(a x) \gamma(p+1, b x) e^{b x} d x=\frac{1}{b} \Gamma(p+1) \sum_{m=0}^{\infty} \frac{1}{(m+p+2)}\left(\frac{b}{a}\right)^{m+p+2}$

$$
=\frac{1}{b} \Gamma(p+1)\left(\frac{b}{a}\right)^{p+2} \Phi\left(\frac{b}{a}, 1, p+2\right) \quad p>-1, a>b
$$

4. $\int_{0}^{\infty} E_{1}(a x) \gamma(n+1, b x) e^{b x} d x=\frac{-n !}{b}\left[\ln \left(\frac{a-b}{b}\right)+\sum_{m=1}^{n-1} \frac{1}{m}\left(\frac{b}{a}\right)^{m}\right] \quad a>b$

5. $\int_{0}^{\infty} x^{p-1} e^{(b-1) x} F_{1}(a ; p ; x) E_{1}(b x) d x=\frac{\Gamma(p) \Gamma(p) \Gamma(1-a)}{b^{p} \Gamma(p+1-a)}$

$$
\times{ }_{2} F_{1}\left(p-a, p ; p-a+1 ; 1-\frac{1}{b}\right) \quad p>0, a<1
$$

6. $\int_{0}^{\infty} x^{p}{ }_{1} F_{1}(2 p+1-a ; 2 p+1 ; x) J_{2 p}(2 \sqrt{b x}) E_{1}(x) d x$

$$
=\frac{\Gamma(2 p+1)}{\Gamma(a)} \cdot b^{p-a} e^{b} \Gamma(a-2 p, b) \gamma(a, b) \quad p>-\frac{1}{2}, a>-1
$$

7. $\int_{0}^{\infty} x^{p-1 / 2} e^{-a x / 2} M_{\kappa, \mu}(a x) E_{1}(b x) d x=\frac{a^{\mu+1 / 2}}{b^{\mu+p+1}} \frac{\Gamma(p+\mu+1)}{(p+\mu+1)}$

$$
\times_{3} F_{2}\left(\frac{1}{2}+\kappa+\mu, p+\mu+1, p+\mu+1 ; 2 \mu+1, p+\mu+2 ;-\frac{a}{b}\right) \quad p+\mu>-1
$$

8. $\int_{0}^{\infty} e^{-(p-1) x} L_{n}(p x) E_{1}(x) d x=\frac{1}{(n+1)}{ }_{2} F_{1}(1, n+1 ; n+2 ; 1-p) \quad 0<p<2$

9. $\int_{0}^{\infty} L_{n}(x) E_{1}(a x) d x=\frac{1}{(n+1)}\left[1-\left(1-\frac{1}{a}\right)^{n+1}\right]$

10. $\int_{0}^{\infty} \operatorname{si}(b x) E_{1}(a x) d x=-\frac{1}{a} \tan ^{-1}\left(\frac{a}{b}\right)-\frac{1}{2 b} \ln \left(1+\frac{b^{2}}{a^{2}}\right)$

11. $\int_{0}^{\infty} C i(a x) E_{1}(a x) d x=-\frac{1}{4 a}(\pi+2 \ln 2)$

12. $\int_{0}^{\infty} \operatorname{erf}(\sqrt{b x}) E_{1}(a x) d x=\frac{1}{a}\left(1+\frac{a}{b}\right)^{1 / 2}+\frac{1}{2 b} \ln \left(\frac{\sqrt{a+b}-\sqrt{b}}{\sqrt{a+b}+\sqrt{b}}\right)$

13. $\int_{0}^{\infty} \operatorname{erfc}\left(\frac{b}{2 \sqrt{x}}\right) E_{1}\left(a^{2} x\right) d x=\frac{1}{a^{2}}(1-a b) e^{-a b}+b^{2} E_{1}(a b)$

14. $\int_{0}^{\infty} \operatorname{erfc}(a x) E_{1}\left(\frac{b^{2}}{x^{2}}\right) \frac{d x}{x^{3}}=\frac{1}{2 b^{2}}(1-2 a b) e^{-2 a b}+2 a^{2} E_{1}(2 a b)$

15. $\int_{0}^{\infty} \operatorname{erfc}(a x) E_{1}\left(\frac{b^{2}}{x^{2}}\right) \frac{d x}{x}=\left[\zeta(2)+(\gamma+\ln 2 a b)^{2}\right]+2 \sum_{m=1}^{\infty} \frac{(-2 a b)^{m}}{m ! m^{2}}$

\subsection{Miscellaneous Integrals}

1. $\int_{0}^{\infty}\left(1-b e^{-p x}\right)^{-1} E_{1}(a x) d x=\frac{1}{a}+\frac{1}{p} \sum_{m=1}^{\infty} \frac{b^{m}}{m} \ln \left(1+\frac{m p}{a}\right) \quad-1 \leqslant b<1$ 
2. $\int_{0}^{\infty}\left(1+b e^{p x}\right)^{-1} E_{1}(a x) d x=-\frac{1}{p} \sum_{m=1}^{\infty} \frac{(-1)^{m}}{b^{m} m} \ln \left(1+\frac{m p}{a}\right) \quad b>1$

3. $\int_{0}^{\infty} \operatorname{sech} x E_{1}(a x) d x=2 \sum_{m=0}^{\infty} \frac{(-1)^{m}}{(2 m+1)} \ln \left(1+\frac{2 m+1}{a}\right)$

4. $\int_{0}^{\infty} \tanh x E_{1}(a x) d x=\frac{1}{a}+\sum_{m=1}^{\infty} \frac{(-1)^{m}}{m} \ln \left(1+\frac{2 m}{a}\right)$

5. $\int_{0}^{\infty} \ln (\cosh x) E_{1}(a x) d x=\frac{3}{2 a^{2}}-\frac{2}{a} \ln 2-\sum_{m=1}^{\infty} \frac{(-1)^{m}}{m^{2}} \ln \left(1+\frac{2 m}{a}\right)$

In $6-8$, let $F(p, q)=\int_{2 \sqrt{a b}}^{\infty} K_{q}(x) \frac{d x}{x^{p}}$

6. $\int_{0}^{\infty} x^{p-1} e^{-b / x} E_{1}(a x) d x=4(2 b)^{p} F(p+1, p)$

7. $\int_{0}^{\infty} \Gamma(p, b / x) E_{1}(a x) d x=b 2^{4-p} F(3-p, p)$

8. $\int_{0}^{\infty} E_{1}(b / x) E_{1}(a x) d x=16 b F(3,0)$

In 9-12, let $G(p, q)=\int_{a b}^{\infty} e^{x} K_{q}(x) \frac{d x}{x^{p}}$

9. $\int_{0}^{\infty}[x(x+2 b)]^{p-1 / 2} E_{1}(a x) d x=\frac{\left(2 b^{2}\right)^{p}}{\sqrt{\pi}} \Gamma\left(p+\frac{1}{2}\right) G(p+1, p) \quad p>-\frac{1}{2}$

10. $\int_{0}^{\infty} \frac{(x+b)}{\sqrt{x(x+2 b)}} E_{1}(a x) d x=b G(1,1)$

11. $\int_{0}^{\infty}\left[(\sqrt{x+2 b}+\sqrt{x})^{2 p}-(\sqrt{x+2 b}-\sqrt{x})^{2 p}\right] E_{1}(a x) d x=p(2 b)^{p+1} G(2, p) \quad p>0$

12. $\int_{0}^{\infty}[x(x+2 b)]^{-p / 2} P_{q}^{p}\left(1+\frac{x}{b}\right) E_{1}(a x) d x=\sqrt{\frac{2}{\pi}} b^{1-p} G\left(\frac{3}{2}-p, \frac{1}{2}+q\right)$

\section{References}

[1] Bierens de Haan, D., Nouvelles Tables d'Intégrales Définies, Amsterdam, 1867.

[2] Bock, P., Composito Math. 7, 123 (1939).

[3] Busbridge, I. W., Quarterly Journal of Mathematics, Oxford Series (2), Vol. 1, 1950.

[4] Erdélyi, A., W. Magnus, F. Oberhettinger, and F. G. Tricomi, Higher Transcendental Functions, Vols. I, II (McGrawHill Book Co., New York, 1953-55; Tables of Integral Transforms, Vols. I, II, McGraw-Hill Book Co., New York, 1954).

[5] Geller, M., A Table of Integrals Involving Powers, Exponentials, Logarithms, and the Exponential Integral, JPL Tech. Rep. 32-469 (Aug. 1963).

[6] Gradshteyn, I. S., and I. M. Ryzhik, Table of Integrals, Series and Products (Academic Press, New York, 1965).

[7] Grobner, W., and N. Hofreiter, Integraltafel, Wien (Springer-Verlag, 1961).

[8] Handbook of Mathematical Functions, edited by M. Abramowitz and I. A. Stegun, National Bureau of Standards, AMS 55, Washington, D.C. (1964).

[9] Hopf, E., Mathematical Problems of Radiative Equilibrium, Cambridge Tracts in Math. and Math. Physics, No. 37 (Cambridge University Press, Cambridge, England, 1934).

[10] Jahnke, E., and F. Emde, Tables of Functions (Dover Publishers, New York, 1945).

[11] Kotani, M., A. Amemiya, E. Ishiguro, and T. Kimura, Table of Molecular Integrals (Maruzen Co., Ltd., Tokyo, Japan, 1955).

[12] Kourganoff, V., Ann. d'Astrophys. 10, 282, 329 (1947); 11, 163 (1948); Comp. Rend. Acad. Sci., Paris 225, 451 (1947); Basic Methods in Transfer Problems (Oxford University Press, London, England, 1952). 
[13] LeCaine, J., A Table of Integrals Involving the Functions $E_{n}(x)$, Chalk River, Ontario, Nat. Res. Council \# 1553 (1948).

[14] McLachlan, N. W., P. Humbert, and L. Poli, Mem. des Sciences Math. L'Acad. des Sciences de Paris, Fasc. 100 (1947), Fasc. 113 (1950).

[15] Nielsen, N., Handbuch der Theorie der Gamma Function, Theorie des Integrallogarithmus and Verwandten Transcendenten, B. G. Teubner, Leipzig, 1906.

(Paper 73B3-299) 